\title{
Curve progression after decompression surgery in patients with mild degenerative scoliosis
}

\author{
Clinical article
}

\author{
Naobumi Hosogane, M.D., Ph.D., ${ }^{1}$ Kota Watanabe, M.D., Ph.D., ${ }^{2}$ \\ Hitoshi Kono, M.D., Ph.D., ${ }^{3}$ Masashi Saito, M.D., ${ }^{3}$ Yoshiaki Toyama, M.D., Ph.D., ${ }^{1}$ \\ and Morio Matsumoto, M.D., Ph.D. ${ }^{1}$
}

${ }^{\prime}$ Orthopedic Surgery and ${ }^{2}$ Department of Advanced Therapy for Spine and Spinal Cord Disorders, Keio University School of Medicine, Shinjuku, Tokyo; and ${ }^{3}$ Spine Center, Keiyu Orthopedic Hospital, Tatebayashi, Gunma, Japan

\begin{abstract}
Object. The authors undertook this study to evaluate curve progression, risk factors for curve progression, and outcomes after decompression surgery in patients with degenerative lumbar scoliosis with minimal to moderate curvature.

Methods. Of 852 patients with lumbar canal stenosis treated by posterior decompression surgery, 50 patients had a lumbar curve greater than $10^{\circ}$ at final follow-up. These patients were divided into 2 groups according to curve progression during the follow-up period: the $\mathrm{P}$ group (11 patients), with a curve progression of more than $5^{\circ}$, and the NP group (39 patients), with a curve progression of $5^{\circ}$ or less. The authors compared preoperative parameters in these 2 groups to elucidate risk factors associated with curve progression and other surgical outcomes.

Results. The average lumbar curve progression in the total group of 50 patients was $3.4^{\circ} \pm 3.9^{\circ}$ (range $-2.0^{\circ}$ to $22.0^{\circ}$ ). In the $\mathrm{P}$ group the average curve progression was $8.5^{\circ}$, and in the NP group it was $2.0^{\circ}$. Multivariate logistic regression analysis showed no significant association between curve progression and any of the potential risk factors evaluated (including curve magnitude, decompression method, and degenerative intervertebral disc changes). Spur formation, evaluated with the Nathan classification at the concave side of the curve, tended to be greater in the P group, although the difference was not statistically significant. There was no significant difference in revision surgery rate, and none of the patients required arthrodesis due to curve progression. Clinical outcomes, evaluated with the Japanese Orthopaedic Association Back Pain Evaluation Questionnaire and the Scoliosis Research Society 22-question questionnaire, were also similar in the 2 groups.

Conclusions. Surgical outcomes did not deteriorate in the P group. While curve progression after decompression surgery could not be predicted from the preoperative factors considered, spur formation at the concave side of the curve may be a candidate factor. The results of this study indicate that spinal fixation to halt deformity progression is not always necessary if the patient's pathological condition derives mainly from canal stenosis. (http://thejns.org/doi/abs/10.3171/2013.1.SPINE12426)
\end{abstract}

KEY WoRds • degenerative lumbar scoliosis • decompression • curve progression

$\mathrm{S}$ YMPTOMATIC DLS significantly impacts the quality of life in an aging population. The disease is usually defined as a spinal deformity with a Cobb angle of more than $10^{\circ}$ in the coronal plane, occurring in a skeletally mature individual and accompanied by degenerative changes in the spine. ${ }^{1}$ Mild symptoms can be managed conservatively. However, patients with severe back pain, leg pain, neurological deficits, or coronal and sagittal imbalance associated with progression of the curvature, and those whose symptoms are not alleviated by conservative treatment, may need surgical intervention.

\footnotetext{
Abbreviations used in this paper: DLS = degenerative lumbar scoliosis; JOABPEQ = Japanese Orthopaedic Association Back Pain Evaluation Questionnaire; SRS-22 = Scoliosis Research Society 22-question questionnaire.
}

Surgical options for DLS include decompression only, decompression with limited fusion, and decompression with extensive corrective fusion. Factors that should be considered when choosing a surgical method include the patient's age, general medical condition, symptoms, degree of osteoporosis, and expectations. Correction and fusion surgery in DLS has high complication rates, so it is reasonable to choose decompression alone when the patient's primary symptom is neurogenic claudication due to spinal canal stenosis. Decompression alone efficiently alleviates radicular and neurogenic claudication symptoms in patients with a relatively mild deformity, although it sometimes fails due to postoperative curve progression that aggravates the symptoms. Although some studies have reported on the natural course of DLS, curve progression after lumbar decompression surgery has not 


\section{N. Hosogane et al.}

been studied. Therefore, it is difficult to determine whether decompression alone is appropriate, or if it is necessary to perform correction and fusion to avoid curve progression.

In this study, we evaluated curve progression after decompression surgery in DLS patients with minimal to moderate preoperative spinal curvature. Our objective was to reveal risk factors for postoperative curve progression and other surgical outcomes, and to estimate the relevancy of decompression surgery in patients with DLS.

\section{Methods}

This study was approved by the Ethics Committee of Keio University.

Patients in whom the diagnosis of lumbar canal stenosis was confirmed by both MRI findings and existence of leg symptoms and who underwent posterior decompression at more than 1 level as an index surgery between 2005 and 2010 were eligible for this study. Among 852 eligible patients, we reviewed the preoperative and latest coronal standing radiographs in those who were followed for more than 1 year after surgery. Patients with a lumbar curve of more than $10^{\circ}$ at the final follow-up and also with a curve of more than $5^{\circ}$ preoperatively were included in the study. There were 50 patients (18 males, 32 females; mean age $[ \pm \mathrm{SD}]$ at time of surgery $70.2 \pm 5.8$ years, range 58.6-83.0 years) who met all these criteria. The mean follow-up period was $2.8 \pm 1.4$ years (range 1.0-8.0 years). Patients with intolerable back pain due to trunk imbalance $\left(>5 \mathrm{~cm}\right.$ ), a large curve of more than $40^{\circ}$, or foraminal stenosis due to lumbar curvature were not included in this study, as these conditions were considered indications for correction and fusion surgery.

Of the 50 patients who met the study criteria, 34 underwent fenestration by a lumbar spinous process splitting technique ${ }^{14}$ at a mean of 1.7 levels (range 1-3 levels), 8 patients underwent conventional fenestration at a mean of 1.8 levels (range 1-3 levels), and 8 patients underwent conventional laminectomy at a mean of 2.3 levels (range 1-4 levels).

Neurological examination findings, preoperative MRI studies, and standing coronal and lateral lumbar radiographs were used to determine each patient's decompression level. Lumbar deformity was not considered in this determination.

Radiographic measurements of deformity were conducted by the first author (N.H.), who was not the primary surgeon and who had 15 years' experience using standardized manual measurement techniques ${ }^{7}$ with standing lumbar coronal and lateral radiographs. Coronal plane parameters included the level of the major curve, the curve magnitude as determined by the Cobb angle, and the tilt angles of L-5 and the pelvis relative to a horizontal line. The curve magnitude within the decompression level was also measured at specific time points. Apical vertebral rotation was assessed by the Nash and Moe classification. ${ }^{6}$ The degree of osteophyte formation of the vertebral body between the upper and lower end vertebrae of the main curve was graded according to the Nathan classification. On coronal radiographs, the osteophytes of all vertebral bodies included in the curve were graded and summed separately on the concave and convex sides. The sum of the Nathan scores on each side was then divided by the number of vertebrae included in the main curve to obtain the average score per level. On the sagittal plane, lumbar lordosis was measured from T-12 to S-1.

Disc degeneration was graded according to the 4-grade classification system described by Schneiderman et al. ${ }^{11}$ Grade 1 (normal) shows normal signal height and intensity; Grade 2 (intermediate) shows a speckled pattern or heterogeneous decreased signal intensity; Grade 3 (marked) has a diffuse loss of signal; and Grade 4 (absent), is signal void. The discs cephalad and caudal to the apex vertebra were graded from preoperative T2-weighted sagittal MRI scans. The sum of the grades of these 2 intervertebral discs was used to assess the degree of disc degeneration in each patient. Clinical outcome was evaluated at the final follow-up using the Japan Orthopaedic Association Back Pain Evaluation Questionnaire (JOABPEQ) and the SRS-22 questionnaire.

Statistical analysis was conducted using an unpaired t-test and a chi-square test. Statistical differences in clinical outcomes were evaluated with the Mann-Whitney Utest. A p value $<0.05$ was considered to be statistically significant. Univariate and multivariate logistic regression analyses were performed to evaluate the odds ratio (with $95 \%$ confidence interval) for potential risk factors for curve progression using SPSS version 19.

\section{Results}

\section{Patient Characteristics and Cobb Angle Progression}

The average progression of the lumbar Cobb angle during the follow-up period was $3.4^{\circ} \pm 3.9^{\circ}$ (range $-2.0^{\circ}$ to $22.0^{\circ}$ ). The preoperative Cobb angle was $16.9^{\circ} \pm 7.3^{\circ}$ $\left(5^{\circ}-40^{\circ}\right)$. We divided the patients into 2 groups according to the degree of curve progression during the follow-up period: the 11 patients with a curve progression of more than $5^{\circ}$ were assigned to the $\mathrm{P}$ group, and the 39 patients with a curve progression of $5^{\circ}$ or less were assigned to the NP group. The NP group was defined as having a curve progression of $5^{\circ}$ or less because there could be a measurement error up to $5^{\circ}$. We compared various parameters in these 2 groups to assess risk factors for curve progression.

Comparison of the 2 groups showed no statistically significant differences in sex, age at surgery, duration of the follow-up period, the number of levels at which decompression was performed, or the decompression method (Table 1).

The Cobb angle of the lumbar curve was $16.1^{\circ}$ in the $\mathrm{P}$ group and $17.2^{\circ}$ in the NP group before surgery and progressed to $24.6^{\circ}$ (progression range $6^{\circ}-22^{\circ}$ ) in the $\mathrm{P}$ group and $19.0^{\circ}$ (progression range $-2^{\circ}$ to $5^{\circ}$ ) in the NP group. There was a significant difference in the degree of progression of the lumbar Cobb angle $\left(8.5^{\circ}\right.$ in the $\mathrm{P}$ group vs $2.0^{\circ}$ in the NP group, $\left.\mathrm{p}<0.05\right)$. The Cobb angle within the decompression levels was $6.9^{\circ}$ in the $\mathrm{P}$ group and $8.1^{\circ}$ in the NP group before surgery and progressed to $13.1^{\circ}$ in the $\mathrm{P}$ group and $8.9^{\circ}$ in the NP group at the final follow-up. The increase in curve magnitude within 


\section{Decompression surgery for degenerative lumbar scoliosis}

the decompression levels was also significantly larger in the $\mathrm{P}$ group than in the $\mathrm{NP}$ group ( $\mathrm{P}$ group $6.2^{\circ}$ vs $\mathrm{NP}$ group $\left.0.8^{\circ}, \mathrm{p}<0.05\right)$.

\section{Preoperative Radiological Parameters}

We evaluated preoperative parameters to identify risk factors for the progression of scoliosis after decompression surgery. Prior to surgery, there were no significant differences in the lumbar Cobb angle or Cobb angle at the decompression level (Table 1).

In the $\mathrm{P}$ group, the apex of the lumbar curve was located at L-2 in 3 patients, L-3 in 4 patients, and L-4 in 4 patients. In the NP group, it was located at L-1 in 1 patient, L-2 in 13 patients, L-3 in 19 patients, and L-4 in 6 patients. The apex of the lumbar curve was included in the decompression level in 9 patients $(81.8 \%)$ and proximal to the decompression level in 2 patients in the $\mathrm{P}$ group. In the NP group, the apex was included in the decompression level in 27 patients $(69.2 \%)$; it was located proximal to the decompression level in 11 patients and distal to the decompression level in 1 patient. Subluxation of more than $5 \mathrm{~mm}$ in the coronal plane was observed preoperatively in 4 patients $(36.4 \%$, mean $7.6 \pm 4.1 \mathrm{~mm})$ in the $\mathrm{P}$ group and 18 patients $(46.2 \%$, mean $9.7 \pm 3.1 \mathrm{~mm})$ in the NP group. Also, spondylolisthesis more than $5 \mathrm{~mm}$ was observed preoperatively in 5 patients $(45.5 \%$, mean $6.4 \pm$ $2.2 \mathrm{~mm})$ in the $\mathrm{P}$ group and 16 patients $(41.0 \%$, mean 6.4 $\pm 2.7 \mathrm{~mm}$ ) in the NP group.

The mean Nash and Moe grade was $1.2 \pm 1.0$ in the $\mathrm{P}$ group and $1.2 \pm 0.7$ in the NP group (not significantly different, Table 2).

The average Nathan classification score of the main curve tended to be higher in the NP group, especially on the concave side, although the difference was not statistically significant (Table 2 ).

The degree of intervertebral disc degeneration at the cephalad and caudal levels was also similar in both groups.

There was no statistically significant difference in lumbar lordosis (assessed by the T12-S1 angle), thoracolumbar alignment (assessed by the T10-L2 angle), L-5 tilt, or pelvic tilt between the 2 groups (Table 2).

Univariate logistic regression analysis was performed with lumbar curve progression as the dependent variable and with the other parameters as independent variables (Table 3). To clarify the risk factors for curve progression, multivariate logistic regression analysis was performed with 4 variables that showed $\mathrm{p}<0.25$ in univariate logistic regression analysis (vertebral osteophyte on the concave side, decompression with fenestration with spinous process splitting, age, and disc degeneration) and the variables that might be clinically important factors for the progression, such as the preoperative Cobb angle of the main curve. Although no variable reached statistical significance, the vertebral osteophyte on the concave side of the curve showed a trend toward significance $(\mathrm{p}=$ 0.062) (Table 4).

\section{Revision Surgery and Clinical Outcomes}

Five patients $(10.0 \%)-1$ in the $\mathrm{P}$ group $(9.0 \%)$ and 4 in the NP group (10.3\%) - underwent revision surgery
TABLE 1: Comparison of demographic and clinical characteristics of 50 patients treated with decompression for DLS*

\begin{tabular}{|c|c|c|c|}
\hline Characteristic & P Group & NP Group & $\begin{array}{c}\mathrm{p} \\
\text { Value }\end{array}$ \\
\hline mean age at surgery (yrs) & $72.8 \pm 6.6$ & $69.5 \pm 5.4$ & NS \\
\hline sex ratio $(M / F)$ & $3: 8$ & $15: 24$ & NS \\
\hline mean follow-up period (yrs) & $3.2 \pm 1.5$ & $2.7 \pm 1.4$ & NS \\
\hline mean no. of levels treated & $1.8 \pm 0.7$ & $1.8 \pm 0.8$ & NS \\
\hline \multicolumn{4}{|l|}{ decompression method } \\
\hline $\begin{array}{l}\text { fenestration w/ spinous process } \\
\text { splitting }\end{array}$ & 3 & 31 & \\
\hline conventional fenestration & 8 & 0 & \\
\hline laminectomy & 0 & 8 & \\
\hline \multicolumn{4}{|l|}{ mean Cobb angle of main curve $\left({ }^{\circ}\right)$} \\
\hline preop & $16.1 \pm 11.2$ & $17.2 \pm 6.0$ & NS \\
\hline final follow-up & $24.6 \pm 10.4$ & $19.0 \pm 6.6$ & $<0.05$ \\
\hline progression & $8.5 \pm 4.8$ & $2.0 \pm 1.9$ & $<0.05$ \\
\hline \multicolumn{4}{|l|}{$\begin{array}{l}\text { mean Cobb angle at decompression } \\
\text { level }\left({ }^{\circ}\right)\end{array}$} \\
\hline preop & $6.9 \pm 3.2$ & $8.1 \pm 4.8$ & NS \\
\hline final follow-up & $13.1 \pm 5.8$ & $8.9 \pm 5.4$ & $<0.05$ \\
\hline progression & $6.2 \pm 6.0$ & $0.8 \pm 3.2$ & $<0.05$ \\
\hline
\end{tabular}

* Values are numbers of patients unless otherwise indicated. Mean values are presented \pm SD. Abbreviation: NS $=$ not significant.

after decompression. The rates of revision surgery did not differ significantly between the 2 groups. The patient in the $\mathrm{P}$ group had decompression surgery (laminectomy) because the stenosis recurred at the treated level. In the NP group, 2 patients had decompression surgery (laminectomy and fenestration by a lumbar spinous process splitting technique) at the previously treated level, 1 patient had

TABLE 2: Comparison of preoperative parameters

\begin{tabular}{|c|c|c|c|}
\hline Parameter & P Group & NP Group & $\begin{array}{c}p \\
\text { Value }\end{array}$ \\
\hline \multicolumn{4}{|l|}{$\begin{array}{l}\text { apical vertebral rotation (Nash } \\
\quad \& \text { Moe) }\end{array}$} \\
\hline neutral (\%) & 27.3 & 17.9 & NS \\
\hline Grade I (\%) & 36.4 & 43.6 & NS \\
\hline Grade II (\%) & 27.3 & 35.9 & NS \\
\hline Grade III (\%) & 9.0 & 2.6 & NS \\
\hline \multicolumn{4}{|l|}{ vertebral osteophyte (Nathan) } \\
\hline concave side & $1.5 \pm 0.5$ & $1.9 \pm 0.7$ & 0.08 \\
\hline convex side & $1.1 \pm 0.1$ & $1.3 \pm 0.4$ & 0.09 \\
\hline $\begin{array}{l}\text { disc degeneration (Schneider- } \\
\text { man) }\end{array}$ & $6.7 \pm 1.2$ & $7.3 \pm 1.3$ & NS \\
\hline lumbar lordosis, T12-S1 $\left(^{\circ}\right)$ & $-33.5 \pm 17.5$ & $-36.9 \pm 13.7$ & NS \\
\hline T10-L2 sagittal angle $\left(^{\circ}\right)$ & $5.2 \pm 10.0$ & $10.1 \pm 12.8$ & NS \\
\hline L-5 tilt angle $\left(^{\circ}\right)$ & $2.1 \pm 8.4$ & $-0.1 \pm 7.0$ & NS \\
\hline pelvic tilt angle $\left({ }^{\circ}\right)$ & $0.8 \pm 2.9$ & $1.2 \pm 2.5$ & NS \\
\hline
\end{tabular}


TABLE 3: Results of univariate logistic regression analysis for risk of progression of lumbar curve

\begin{tabular}{|c|c|c|c|}
\hline Variable & OR & $95 \% \mathrm{Cl}$ & $\begin{array}{c}\mathrm{p} \\
\text { Value }\end{array}$ \\
\hline \multicolumn{4}{|l|}{ vertebral osteophyte (Nathan) } \\
\hline concave side & 0.308 & $0.081-1.173$ & 0.084 \\
\hline convex side & 0.041 & $0.001-2.216$ & 0.117 \\
\hline \multicolumn{4}{|l|}{ decompression method } \\
\hline conventional fenestration & 1 & - & - \\
\hline $\begin{array}{l}\text { fenestration w/ spinous process } \\
\text { splitting }\end{array}$ & 0.222 & $0.038-1.306$ & 0.096 \\
\hline laminectomy & 0.800 & $0.101-6.437$ & 0.833 \\
\hline age & 1.109 & $0.978-1.258$ & 0.106 \\
\hline disc degeneration (Schneiderman) & 0.728 & $0.442-1.198$ & 0.211 \\
\hline L-5 tilt angle & 1.062 & $0.959-1.177$ & 0.250 \\
\hline follow-up period & 1.234 & $0.790-1.930$ & 0.356 \\
\hline $\begin{array}{l}\text { decompression level including apex } \\
\text { of curve }\end{array}$ & 2.000 & $0.374-10.691$ & 0.418 \\
\hline $\begin{array}{l}\text { apical vertebral rotation (Nash \& Moe } \\
\text { grade) }\end{array}$ & 1.689 & $0.422-6.763$ & 0.459 \\
\hline $\begin{array}{l}\text { Cobb angle at decompression level } \\
\text { (preop) }\end{array}$ & 0.946 & $0.809-1.105$ & 0.482 \\
\hline lumbar lordosis (T12-S1) & 1.016 & $0.970-1.065$ & 0.492 \\
\hline T10-L2 sagittal angle & 0.980 & $0.923-1.041$ & 0.521 \\
\hline rotatory subluxation $>5 \mathrm{~mm}$ (preop) & 0.667 & $0.168-2.651$ & 0.565 \\
\hline Cobb angle of main curve (preop) & 0.979 & $0.888-1.079$ & 0.668 \\
\hline pelvic tilt angle & 0.955 & $0.696-1.311$ & 0.776 \\
\hline spondylolisthesis $>5 \mathrm{~mm}$ (preop) & 1.198 & $0.311-4.609$ & 0.793 \\
\hline
\end{tabular}

decompression surgery (fenestration by a lumbar spinous process splitting technique) at the cephalad adjacent level, and 1 patient had posterior lumbar interbody fusion due to foraminal stenosis at the decompressed level. None of the patients in either group required multilevel correction and fusion surgery due to scoliosis progression.

Clinical outcomes at final follow-up are shown in Fig. 1. The patients' walking ability and social life function, measured by JOABPEQ, tended to be lower in the P than the NP group, although the differences were not statistically significant. The SRS-22 scores were similar in the 2 groups. Visual analog scale scores for low back pain, leg pain, or leg numbness showed no significant differences between the 2 groups (data not shown).

\section{Discussion}

\section{Decompression Surgery for $D L S$}

Surgical options for DLS include decompression only, decompression with limited fusion, or decompression with extensive correction and fusion. Several factors must be considered when choosing the optimal surgical method. Surgical treatment for adult scoliosis has a high incidence of complications. A systematic literature review by Yadla et al. ${ }^{15}$ showed an incidence of perioperative adverse events of more than $40 \%$. Therefore, surgical invasion should be minimized, especially for elderly patients
TABLE 4: Results of multivariate logistic regression analysis for risk of progression of lumbar curve

\begin{tabular}{lccc}
\hline \multicolumn{1}{c}{ Variable } & OR & $95 \% \mathrm{Cl}$ & $\mathrm{p} \mathrm{Value}$ \\
\hline $\begin{array}{l}\text { vertebral osteophyte (Nathan) of con- } \\
\text { cave side }\end{array}$ & 0.215 & $0.43-1.063$ & 0.062 \\
$\begin{array}{l}\text { decompression method fenestration } \\
\text { w/ spinous process splitting) }\end{array}$ & - & - & 0.143 \\
age & - & - & 0.296 \\
Cobb angle of main curve (preop) & - & - & 0.459 \\
disc degeneration (Schneiderman) & & & 0.500 \\
\hline
\end{tabular}

with comorbidities. Transfeldt et al. ${ }^{12}$ compared outcomes in patients treated with decompression alone, decompression with limited fusion, or decompression and full curve fusion, and found that the group treated with decompression alone had the lowest rate of complications. However, the indications for decompression surgery for DLS are still controversial.

Several studies have discussed the indications for decompression surgery alone in patients with DLS. Postacchini $^{8}$ suggested that elderly patients who present with mild scoliosis and little or no back pain can be managed with decompressive surgery alone. Gupta ${ }^{2}$ reported that decompression surgery alone can be performed in patients with minor curves (less than $30^{\circ}$ ) and 1 to 2 levels of stenosis with minimal rotary or lateral listhesis (2-3 $\mathrm{mm}$ ); males with large osteophytes, which render the spine stable, can tolerate a laminectomy of more than 2 levels without fusion. Aebi ${ }^{1}$ suggested that decompression may be performed if symptoms are limited to the legs and there is no relevant back pain; however, fixation should be considered if decompression is performed at the apex or at the bottom of the curve. Vaccaro and Ball ${ }^{13}$ recommended fusion surgery for DLS because decompression without fusion often aggravates the collapse of a degenerative curve, causing increased instability, continued back pain, and exacerbated neural symptoms.

\section{Curve Progression After Decompression in DLS}

One of the reasons a surgeon may hesitate to perform decompression-only surgery is the lack of data on curve progression after decompression surgery in patients with or without scoliosis. In this study, we evaluated curve progression after decompression surgery in DLS patients with a minimal to moderate curve, and observed a mean curve progression of $3.4^{\circ}$ during a mean follow-up period of 2.8 years. The average progression of the curve was $1.4^{\circ}$ per year for all patients, and the curve progressed more than $5^{\circ}$ in $21.6 \%$ of the patients.

Studies have found similar rates of curve progression in the natural course of DLS. For example, Kluba et al. ${ }^{5}$ found a mean Cobb angle increase of $2.8^{\circ}$ during an average follow-up period of 3.8 years. Robin et al ${ }^{10}$ found that scoliosis increased over $4^{\circ}$ in $46 \%$ of 544 patients followed for $7-13$ years. The average progression was $7^{\circ}$ in patients with a Cobb angle greater than $10^{\circ}$. Pritchett et al..$^{9}$ observed an average $3^{\circ}$ of progression per year in $73 \%$ of patients followed over a 5-year period. Thus, the 

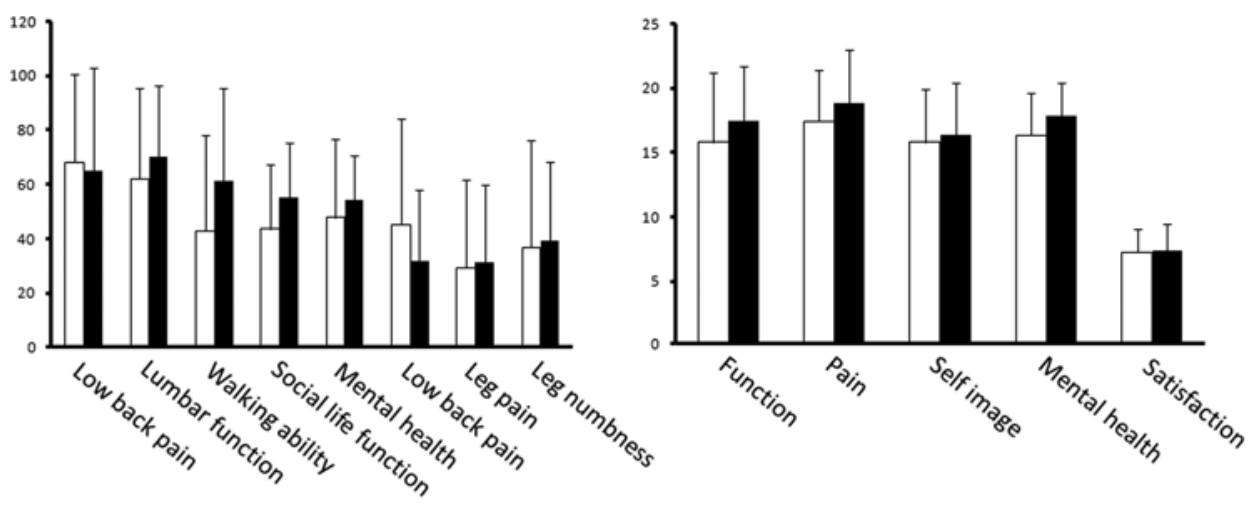

FIG. 1. Clinical outcomes at final follow-up. Left: Results of JOABPEQ. Right: Results of SRS-22. White bars indicate the $P$ group and black bars the NP group.

curve progression after decompression surgery is almost equivalent to that occurring in the natural course of DLS.

\section{Risk Factor for Curve Progression}

Our study found no significant differences between the $\mathrm{P}$ and NP groups in the preoperative parameters of age, Cobb angle, apical rotation, location of the apex relative to the decompression level, rotatory subluxation, spondylolisthesis, or disc degeneration. Also there was no significant risk factor identified with multivariate logistic regression analysis, although spur formation on the concave side of the curve, which might stabilize the spine, may be a candidate factor for reduced risk of curve progression.

\section{Reoperation After Decompression}

Reoperation rates after decompression surgery for spinal stenosis vary from $5 \%$ to $23 \%$. Hansraj et al. ${ }^{3}$ reported that only $5 \%$ of patients required reoperation during a 5-year follow-up period and suggested that decompression surgery is indicated only for typical spinal stenosis, defined as scoliosis of less than $20^{\circ}$ or without instability. Katz et al. ${ }^{4}$ reported that $23 \%$ of patients required revision surgery 7 to 10 years after decompression for lumbar canal stenosis. Of those who underwent revision surgery, $35 \%$ had arthrodesis due to instability, $20 \%$ had laminectomies at adjacent levels, and 25\% had laminectomies at the same level. In our study, 5 patients $(10.0 \%)$ required revision surgery during an overall mean follow-up period of 2.8 years. Of these, 3 patients $(60 \%)$ had decompression at the same level, 1 (20\%) had decompression at the adjacent level, and 1 (20\%) had interbody fusion. The reoperation rates in our study did not differ significantly from those in previous reports. The patients who required revision surgery in our study had recurrent leg pain due either to restenosis at the treated level or to newly developed stenosis at the adjacent level. We did not find any association between reoperation and the progression of scoliosis after the initial surgery, and none of the patients underwent reoperation due to symptomatic curve progression of the scoliosis. Therefore, decompression surgery seems appropriate for patients whose chief complaint is leg pain and who do not have low back pain from coronal or sagittal imbalances.
This study is the first to evaluate curve progression after decompression surgery. However, it has several limitations. First, this was not a randomized study, and we were unable to define the optimal indication for decompression surgery (decompression alone) for patients with DLS. Although the patients in this study had DLS with a minimal to moderate preoperative curve, this study provides information about curve progression after decompression surgery that might assist a surgeon in determining an appropriate surgical method. Second, we do not have baseline (preoperative) values for the questionnaires used to assess patients' symptoms (the JOABPEQ and the SRS-22). In the case of the JOABPEQ, this is because the questionnaire has only been available since 2009, when most of the patients had already undergone surgery. In the case of the SRS-22, it is because this questionnaire evaluates symptoms due to deformity, and we do not routinely use it for patients with lumbar canal stenosis. Finally, we were not able to identify significant risk factors for curve progression based on preoperative data, possibly due to the small sample size. We have performed power analysis using $G^{*}$ Power 3.1 (software freely available on the Internet). We would need approximately 40 patients in the $\mathrm{P}$ group and 120 patients in the NP group to obtain sufficient power such as 0.8 , an effect size of 0.5 , and an $\alpha$ error probability of 0.05 . However, because the average progression of the curve was only $3.4^{\circ}$ during 2.8 years of follow-up, further study based on a larger sample size (an estimated size of more than 2500 patients with lumbar canal stenosis) and longer follow-up period should be performed to confirm our results.

\section{Conclusions}

This study clarified that both the frequency and degree of curve progression after decompression surgery for DLS patients with a minimal to moderate preoperative curve are equivalent to those occurring in the natural course of DLS. In addition, this study did not find a significant increase in reoperation rates compared with previously reported rates for decompression surgery for lumbar canal stenosis, and no patient required arthrodesis due to curve progression. These results indicate that spinal correction and fusion to halt progression of deformity is not always necessary if the patient's pathological 


\section{N. Hosogane et al.}

condition derives mainly from spinal canal stenosis. Our study also indicates that while preoperative prediction of curve progression after decompression surgery is difficult, spur formation on the concave side of the curve may be a predictive factor.

\section{Disclosure}

The authors report no conflict of interest concerning the materials or methods used in this study or the findings specified in this paper.

Author contributions to the study and manuscript preparation include the following. Conception and design: Hosogane. Acquisition of data: Hosogane. Analysis and interpretation of data: Hosogane, Kono, Saito. Drafting the article: Hosogane. Critically revising the article: all authors. Reviewed submitted version of manuscript: all authors. Approved the final version of the manuscript on behalf of all authors: Matsumoto. Statistical analysis: Hosogane. Study supervision: Matsumoto, Kono, Saito, Toyama.

\section{References}

1. Aebi M: The adult scoliosis. Eur Spine J 14:925-948, 2005

2. Gupta MC: Degenerative scoliosis. Options for surgical management. Orthop Clin North Am 34:269-279, 2003

3. Hansraj KK, Cammisa FP Jr, O'Leary PF, Crockett HC, Fras CI, Cohen MS, et al: Decompressive surgery for typical lumbar spinal stenosis. Clin Orthop Relat Res (384):10-17, 2001

4. Katz JN, Lipson SJ, Chang LC, Levine SA, Fossel AH, Liang MH: Seven- to 10-year outcome of decompressive surgery for degenerative lumbar spinal stenosis. Spine (Phila Pa 1976) 21:92-98, 1996

5. Kluba T, Dikmenli G, Dietz K, Giehl JP, Niemeyer T: Comparison of surgical and conservative treatment for degenerative lumbar scoliosis. Arch Orthop Trauma Surg 129:1-5, 2009

6. Nash CL Jr, Moe JH: A study of vertebral rotation. J Bone Joint Surg Am 51:223-229, 1969

7. O'Brien MF, Kukol TR, Blanke KM, Lenke LG (eds): Spi- nal Deformity Study Group Radiographic Measurement Manual. Memphis, TN: Medtronic Sofamor Danek, 2004

8. Postacchini F: Surgical management of lumbar spinal stenosis. Spine (Phila Pa 1976) 24:1043-1047, 1999

9. Pritchett JW, Bortel DT: Degenerative symptomatic lumbar scoliosis. Spine (Phila Pa 1976) 18:700-703, 1993

10. Robin GC, Span Y, Steinberg R, Makin M, Menczel J: Scoliosis in the elderly: a follow-up study. Spine (Phila Pa 1976) 7:355-359, 1982

11. Schneiderman G, Flannigan B, Kingston S, Thomas J, Dillin WH, Watkins RG: Magnetic resonance imaging in the diagnosis of disc degeneration: correlation with discography. Spine (Phila Pa 1976) 12:276-281, 1987

12. Transfeldt EE, Topp R, Mehbod AA, Winter RB: Surgical outcomes of decompression, decompression with limited fusion, and decompression with full curve fusion for degenerative scoliosis with radiculopathy. Spine (Phila Pa 1976) 35: 1872-1875, 2010

13. Vaccaro AR, Ball ST: Indications for instrumentation in degenerative lumbar spinal disorders. Orthopedics 23:260-271, 2000

14. Watanabe K, Hosoya T, Shiraishi T, Matsumoto M, Chiba K, Toyama Y: Lumbar spinous process-splitting laminectomy for lumbar canal stenosis. Technical note. J Neurosurg Spine 3: 405-408, 2005

15. Yadla S, Maltenfort MG, Ratliff JK, Harrop JS: Adult scoliosis surgery outcomes: a systematic review. Neurosurg Focus 28(3):E3, 2010

Manuscript submitted May 1, 2012.

Accepted January 2, 2013.

Portions of this work were presented in abstract form at the International Meeting on Advanced Spine Techniques in Toronto, Canada, July 2010.

Please include this information when citing this paper: published online February 1, 2013; DOI: 10.3171/2013.1.SPINE12426.

Address correspondence to: Morio Matsumoto, M.D., Department of Orthopedic Surgery, Keio University School of Medicine, 35 Shinanomachi, Shinjuku, Tokyo 160-8582, Japan. email: morio@a5.keio.jp. 